\title{
WEAK AMENABILITY OF RIGHT-ANGLED COXETER GROUPS
}

\author{
ALAIN VALETTE
}

(Communicated by Palle E. T. Jorgensen)

\begin{abstract}
We show that the representation-theoretic result of Januszkiewicz has an impact on harmonic analysis and operator algebras; more precisely, rightangled Coxeter groups are weakly amenable with Cowling-Haagerup constant 1 ; as a consequence, von Neumann algebras with Cowling-Haagerup constant $>1$ are not embeddable into the von Neumann algebra of a right-angled Coxeter group.
\end{abstract}

This is an appendix to T. Januszkiewicz's paper For right-angled Coxeter groups $z^{|g|}$ is a coefficient of a uniformly bounded representation [Ja].

For a locally compact group $G$, we denote by $A(G)$ the Fourier algebra of $G$ and by $M_{0} A(G)$ the algebra of completely bounded multipliers of $A(G)$. We say that $G$ is weakly amenable in the sense of Cowling and Haagerup $[\mathrm{CH}]$ if there exists a number $M \geq 1$ and a net $\left(\psi_{i}\right)_{i \in I}$ in $A(G)$ such that

(i) $\left\|\psi_{i}\right\|_{M_{0} A(G)} \leq M$ for all $i \in I$;

(ii) $\left(\psi_{i}\right)_{i \in I}$ converges to 1 uniformly on compact subsets of $G$.

The $C H$-constant $\Lambda_{G}$ is the infimum of all $M$ 's for which such a net $\left(\psi_{i}\right)_{i \in I}$ can be found. The aim of this note is to deduce from [Ja] the following:

Theorem. Let $\Gamma$ be a right-angled Coxeter group; then $\Gamma$ is weakly amenable, with $C H$-constant $\Lambda_{\Gamma}=1$.

The Theorem follows immediately from the following Proposition, by applying it to the holomorphic family $\left(\pi_{z}\right)_{z \in D}$ of uniformly bounded representations of $\Gamma$ constructed in [Ja], and to the word length function $L: \Gamma \rightarrow \mathbb{N}: g \rightarrow$ $L(g)=|g|$. Here and below, $D$ denotes the open unit disk in $\mathbb{C}$.

Proposition. Let $G$ be a locally compact group, endowed with a proper continuous function $L: G \rightarrow \mathbb{N}$ which is zero at $1 \in G$. Assume that there exists a holomorphic family $\left(\pi_{z}\right)_{z \in D}$ of uniformly bounded strongly continuous representations of $G$ on a fixed Hilbert space $\mathscr{H}$, with $\pi_{t}$ unitary for $\left.t \in\right] 0,1[$, and a vector $\xi \in \mathscr{H}$ such that, for any $z \in D$ and $g \in G$,

$$
z^{L(g)}=\left\langle\pi_{z}(g) \xi \mid \xi\right\rangle \text {. }
$$

Then $G$ is weakly amenable, with $C H$-constant $\Lambda_{\cdot}=1$.

Received by the editors March 25, 1992.

1991 Mathematics Subject Classification. Primary 22D12, 22D25, 46L10. 
Proof of the Proposition. For $z \in D$, denote by $\psi_{z}$ the function $g \rightarrow z^{L(g)}$ on $G$. Then $z \rightarrow \psi_{z}$ is a holomorphic map from $D$ to $M_{0} A(G)$.

For $N \in \mathbb{N}$, let

$$
F_{N}\left(e^{i \theta}\right)=\sum_{|n| \leq N}\left(1-\frac{|n|}{N+1}\right) e^{i n \theta}=\frac{1-\cos (N+1) \theta}{(N+1)(1-\cos \theta)}
$$

be the Féjer kernel. It has the two following properties:

(a) $F_{N} \geq 0$;

(b) $F_{N}$ is an approximate identity, i.e., for any continuous function $f$ on the circle $S^{1}$,

$$
\lim _{n \rightarrow \infty}(2 \pi)^{-1} \int_{0}^{2 \pi} F_{N}\left(e^{i \theta}\right) f\left(e^{i \theta}\right) d \theta=f(1)
$$

(see [Zy, p. 45] for details). Now, for $0<r<1$, set

$$
\boldsymbol{\Phi}_{N, r}=(2 \pi)^{-1} \int_{0}^{2 \pi} F_{N}\left(e^{i \theta}\right) \psi_{r e^{i \theta}} d \theta .
$$

Then $\Phi_{N, r}$ belongs to $M_{0} A(G)$; using (a), we have

$$
\begin{aligned}
\left\|\Phi_{N, r}-\psi_{r}\right\|_{M_{0} A(G)} & =(2 \pi)^{-1}\left\|\int_{0}^{2 \pi} F_{N}\left(e^{i \theta}\right)\left(\psi_{r e^{i \theta}}-\psi_{r}\right) d \theta\right\|_{M_{0} A(G)} \\
& \leq(2 \pi)^{-1} \int_{0}^{2 \pi} F_{N}\left(e^{i \theta}\right)\left\|\psi_{r e^{i \theta}}-\psi_{r}\right\|_{M_{0} A(G)} d \theta .
\end{aligned}
$$

Appealing to (b), we conclude

$$
\lim _{N \rightarrow \infty}\left\|\Phi_{N, r}-\psi_{r}\right\|_{M_{0} A(G)}=0 .
$$

Now $\left\|\psi_{r}\right\|_{M_{0} A(G)}=1$ because $\pi_{r}$ is unitary. So we have simultaneously

$$
\lim _{N \rightarrow \infty}\left\|\Phi_{N, r}\right\|_{M_{0} A(G)}=1
$$

and

$$
\lim _{r \rightarrow 1} \lim _{N \rightarrow \infty} \Phi_{N, r}=1 \quad \text { uniformly on compact subsets of } G .
$$

Suppose that we know that $\Phi_{N, r}$ belongs to $A(G)$; then it follows straight from the definitions that $\Lambda_{G}=1$. To check that $\Phi_{N, r}$ is in $A(G)$, we compute

$$
\begin{aligned}
\Phi_{N, r}(g) & =(2 \pi)^{-1} \int_{0}^{2 \pi} \sum_{|n| \leq N}\left[1-\frac{|n|}{N+1}\right] e^{i n \theta}\left(r e^{i \theta}\right)^{L(g)} d \theta \\
& = \begin{cases}\left(1-\frac{L(g)}{N+1}\right) r^{L(g)} & \text { if } L(g) \leq N, \\
0 & \text { otherwise. }\end{cases}
\end{aligned}
$$

This shows that $\Phi_{N, r}$ has compact support on $G$. Set $K=\{k \in G: L(k)=0\}$. Fix $r \in] 0,1[$; then

$$
k \in K \Leftrightarrow\left\langle\pi_{r}(k) \xi \mid \xi\right\rangle=1 \Leftrightarrow \pi_{r}(k) \xi=\xi .
$$

This shows that $K$ is a compact open subgroup of $G$. Moreover, for $k \in K$ and $g \in G$,

$$
r^{L\left(k^{-1} g\right)}=\left\langle\pi_{r}\left(k^{-1} g\right) \xi \mid \xi\right\rangle=\left\langle\pi_{r}(g) \xi \mid \pi_{r}(k) \xi\right\rangle=r^{L(g)}
$$


so that $L\left(k^{-1} g\right)=L(g)$. Let $\chi_{K}$ be the characteristic function of $K$; we normalize Haar measure on $G$ in such a way that $K$ has measure 1 . Then, using the fact that $\Phi_{N, r}(g)$ only depends on $L(g)$, we have

$$
\chi_{K} * \boldsymbol{\Phi}_{N, r}(g)=\int_{K} \boldsymbol{\Phi}_{N, r}\left(k^{-1} g\right) d k=\int_{K} \Phi_{N, r}(g) d k=\Phi_{N, r}(g) .
$$

The equality $\chi_{K} * \Phi_{N, r}=\Phi_{N, r}$ shows that $\Phi_{N, r}$ belongs to $A(G)$, as a convolution of two continuous functions with compact support. This completes the proof of the Proposition.

We are grateful to the referee for suggesting the use of the Fejer kernel, allowing us to simplify the proof and to remove a superfluous extra assumption from the original version of the Proposition.

Remark. Let $X$ be a locally finite tree. Fix a vertex $x_{0}$. There is a holomorphic family $\left(\pi_{z}\right)_{z \in D}$ of uniformly bounded representations of Aut $X$ on $l^{2}(X)$, unitary for $t \in] 0,1[$ and such that, for $g \in$ Aut $X$,

$$
\left\langle\pi_{z}(g) \delta_{x_{0}} \mid \delta_{x_{0}}\right\rangle=z^{\left|g x_{0}-x_{0}\right|},
$$

where $\left|g x_{0}-x_{0}\right|$ is the distance between $x_{0}$ and $g x_{0}$ (see [Sz, V1]). The above Proposition then shows that any closed subgroup of Aut $X$ is weakly amenable with $C H$-constant $\Lambda_{G}=1$. For other proofs of this fact, see Theorem 8 in [Sz] and Proposition 8 in [V2]; see also [BP] for important special cases.

For a discrete group $\Gamma$, we denote by $\mathrm{vN}(\Gamma)$ the von Neumann algebra of $\Gamma$, i.e., the bicommutant of the left regular representation of $\Gamma$. For a von Neumann algebra $M$, one may define a $C H$-constant $\Lambda_{M}$ (see [CH, $\left.\S 6\right]$; one has $\Lambda_{\Gamma}=\Lambda_{\mathrm{vN}(\Gamma)}[\mathrm{CH}$, Proposition 6.1]). If $M$ is a von Neumann algebra with a finite faithful trace (this is automatic for group von Neumann algebras) and $N$ is a von Neumann subalgebra, then $\Lambda_{N} \leq \Lambda_{M}$ [CH, Proposition 6.3]. It follows that our theorem has the following immediate consequence:

Corollary. Let $\Gamma$ be a right-angled Coxeter group; let $N$ be a von Neumann algebra with $\Lambda_{N}>1$. Then $N$ is not isomorphic to any von Neumann subalgebra of $\mathrm{vN}(\Gamma)$.

This Corollary has to be compared to the following result (see [De, Theorem 4.11.1]): if $\Gamma$ is any Coxeter group and if $N$ is a $\mathrm{II}_{1}$-factor with property (T) in the sense of Connes-Jones [CJ], then $N$ is not isomorphic to any von Neumann subalgebra of $\mathrm{vN}(\Gamma)$.

We conclude by mentioning three open questions of harmonic analysis on general Coxeter groups; all of them are related to weak forms of amenability, as discussed in [V3]. We conjecture that the answer is affirmative for all.

(1) Are Coxeter groups weakly amenable with $C H$-constant 1 ? In view of the above Proposition, this would be the case if the results of [Ja] are proved for all Coxeter groups.

(2) Are Coxeter groups $K$-amenable in the sense of Cuntz [Cu]? Note that this is the case for a right-angled Coxeter group $\Gamma$; indeed, Pimsner has shown that an amalgamated product of two $K$-amenable groups its itself $K$-amenable [Pi]. Now, we may assume that at least one weight $m_{i j}$ in the Coxeter diagram of $\Gamma$ is $\infty$ (otherwise $\Gamma$ is finite); then $\Gamma$ is an amalgamated product of two right-angled Coxeter groups with less generators than $\Gamma$; an immediate induction gives the result. 
(3) Let $\Gamma$ be a Coxeter group and $n \geq 1$ an integer. Does the reduced $C^{*}$-algebra of $\Gamma$ have the $n$-positive approximation property? This is not even known for right-angled Coxeter groups. (This property is obvious for spherical or affine Coxeter groups - these are amenable-and is proved in [JV] for Coxeter groups that are hyperbolic in Gromov's sense, plus a few examples that are neither spherical, nor affine, nor hyperbolic.)

\section{REFERENCES}

[BP] M. Bozejko and M. Picardello, Weakly amenable groups and amalgamated products, Proc. Amer. Math. Soc. 117 (1993), 1039-1046.

[CJ] A. Connes and V. F. R. Jones, Property (T) for von Neumann algebras, Bull. London Math. Soc. 17 (1985), 57-62.

[CH] M. Cowling and U. Haagerup, Completely bounded multipliers of The Fourier algebra of a simple Lie group of real rank one, Invent. Math. 96 (1989), 507-549.

[Cu] J. Cuntz, K-theoretic amenability for discrete groups, J. Reine Angew. Math. 344 (1983), $180-195$.

[De] A. Deutsch, Kazhdan's property $(\mathrm{T})$ and related properties of loally compact and discrete groups, Ph.D. thesis, Univ. of Edinburgh, 1992.

[Ja] T. Januszkiewicz, For right-angled Coxeter groups $z^{|g|}$ is a coefficient of a uniformly bounded representation, Proc. Amer. Math. Soc. 119 (1993), 1115-1119.

[JV] P. Jolissaint and A. Valette, Normes de Sobolev et convoluteurs bornés sur $L^{2}(G)$, Ann. Inst. Fourier (Grenoble) 41 (1991), 797-822.

[Pi] M. Pimsner, $K K$-groups of crossed products by groups acting on trees, Invent. Math. 86 (1986), 603-634.

[Sz] R. Szwarc, Groups acting on trees and approximation properties of the Fourier algebra, J. Funct. Anal. 95 (1991), 320-343.

[V1] A. Valette, Cocycles d'arbres et représentations uniformément bornées, C. R. Acad. Sci. Paris Sér. I 310 (1990), 703-708.

[V2] _ Les représentations uniformément bornées associées à un arbre réel, Bull. Soc. Math. Belg. 42 (1990), 747-760.

[V3] _ Weak forms of amenability for split rank 1 p-adic groups, $p$-Adic Methods and their Applications (A. J. Baker and R. J. Plymen, eds.), Oxford Science Publ., Clarendon Press, Oxford, 1992, pp. 143-165.

[Zy] A. Zygmund, Trigonometrical series, 2nd ed., Chelsea, New York, 1952.

Institut de MathematiQues, Université de Neuchâtel, Chantemerle 20, CH-2007 NEUCHÂTEL, SUISSE

E-mail address: valette@maths.unine.ch 